\title{
LES SECTEURS CLAIRS ET SOMBRES DES COLONIES DE LEVURES
}

\author{
Par Maurice LANGERON et Paul GUERRA
}

Les colonies géantes de levures et de champignons levuriformes présentent assez fréquemment à leur surface des secteurs dont la coloration claire ou foncée tranche sur le fond général de la colonie. Ces secteurs peuvent être isolés ou au nombre de 2,3 , 4, etc.; ils forment alors une rosace ressemblant à une fleur (fig. 4, pl. IV), surtout lorsqu'ils sont allongés en forme de pétales.

Ces secteurs échappent la plupart du temps à l'observation, parce que les microbiologistes emploient le déplorable procédé de la culture en strie, couvrant toute la surface de la gélose inclinée. Il en résulte que les microcolonies, formant la strie d'ensemencement, fusionnent en quelques heures et n'ont pas le temps de développer leur morphologie macroscopique. On n'a bientòt plus qu'une pâte crémeuse uniforme qui peut se plisser par la suite mais qui ne présente aucune autre différenciation. La strie n'est bonne que pour se procurer rapidement une assez grande quantité de culture jeune et active, en vue d'une épreuve de fermentation ou d'assimilation. Elle est funeste pour l'étude.

Une autre erreur très répandue consiste à n'étudier que de jeunes colonies de 24 ou 48 heures : c'est ainsi que la plupart des figures hâtivement publiées depuis quelques années par les auteurs qui se sont occupés de zymologie médicale ne présentent aucun détail utilisable : ce sont des images banales qu'on rencontre au début du développement de toutes les levures et qui n'ont rien de caractéristique.

Les colonies de levures doivent être conservées et observées pendant des semaines et même des mois : si le milieu est bien préparé, les colonies inférieures conservent leur vitalité, sans se dessécher, pendant 4, 5 ou 6 mois. C'est alors qu'on peut étudier les variantes $S$ et $R$ avec leur alternance et leur réversibilité, ou encore les secteurs qui vont faire l'objet de ce mémoire. Dans les légendes de nos figures, nous donnons autant que possible l'àge de nos cultures.

C'est naturellement chez Candida albicans que nous avons le plus

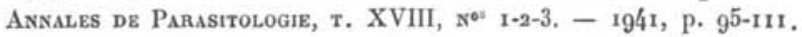


fréquemment observé l'apparition de secteurs, parce que c'est l'espèce la plus répandue et celle dont nous avons pu examiner le plus grand nombre de souches. Mais d'autres espèces présentent la même particularité. Ce sont, jusqu'ici : $C$. triadis, $C$. pseudotropicalis, $C$. guilliermondi, $C$. flareri, $C$. parakrusei, $C$. pelliculosa.

Les secteurs ne sont pas spéciaux aux colonies de levures anascosporées. On les observe aussi chez des levures ascosporées, notamment dans le genre Debaryomyces où ils paraissent fréquents.

Les données bibliographiques concernant cette question sont extrêmement rares, pour ne pas dire inexistantes, à cause des deux erreurs de technique et d'observation que nous venons de signaler. C'est tout au plus si nous avons trouvé une indication de ces secteurs dans l'excellent ouvrage d'Henrici ( $\mathrm{r} 930, \mathrm{p} .205)$ et dans deux mémoires

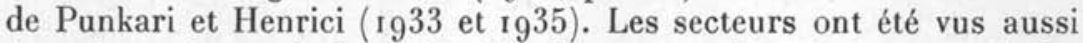
dans des cultures de bactéries (Hadley, I9 19 , dissociation marginale, Ztschr. f. Hyg., LXXXIX, г9r9, p. 339; Gratia, r922, Jl exper. med., XXXV, r922, p. 287 ; A. D. Dulaney, 1928 , dissociation marginale, $J l$. infect. dis., XLII, 1928 , p. $575-588)$ : dans ces trois cas, il s'agit de cultures d'Escherichia coli.

Dans notre mémoire de 1938 (ces Annales, XVI, r938, pp. 36-84, I62-I 79, 429-476, 48I-525, pl. XII-XXXIII), nous avons mentionné l'existence de secteurs clairs ou sombres chez $C$. albicans (p. 443), C. pelliculosa (p. 463), C. guilliermondi (p. 469), C. parakrusei (p. 49r et 493), C. flareri (p. 499), en insistant particulièrement sur C. parakrusei.

\section{I. - Secteurs des colonies de levures anascosporées.}

A. - Secteurs des colonies de Mycotoruloïdées (Candida)

1. Candida guilliermondi. - Nous avons signalé ( 1938, p. 469) l'existence de stries rayonnantes alternativement blanches et sombres, dans nos souches de C. guilliermondi. Nous pouvons en figurer un exemple remarquable fourni par la souche $7_{82}$ B isolée par Rivalier à l'hôpital Saint-Louis, d'une onychomycose (cas Picard) (fig. 4, pl. IV). La netteté des fermentations ne laisse aucun doute sur l'identification de cette souche.

2. Candida pseudotropicalis. - Les secteurs doivent apparaître rarement dans les colonies de cette espèce, car la surface est demitransparente, en glacis, non bombée. Nous arons pourtant rencon- 
tré un bel exemple de secteurs sombres dans une colonie de notre souche 496 B ( 1938 , p. 465), souche précédemment identifiée par la fermentation si caractéristique du lactose (fig. 6, pl. IV), ainsi que dans la souche $768 \mathrm{~B}$ (souche type de Castellani).

3. Candida parakrusei. - Nous avons précédemment décrit (r938, p. 49I, pl. XXXII, fig. 4) les secteurs jaunâtres qui apparaissent fréquemment sur les bords des colonies de cette espèce. Nous avons signalé les énormes cellules, généralement acido-résistantes, qui forment la plus grande partie de ces secteurs, et nous avons figuré les arbuscules et chaînettes si remarquables de ces blastospores géantes (fig. I-3, pl. XVIII, I938). Ces microphotographies, auxquelles nous prions le lecteur de se reporter, montrent les blastospores naissant soit directement d'une microcolonie de blastospores rondes de petite taille, soit d'un point quelconque d'un rameau du type Mycocandida, si caractéristique dans cette espèce ( $\mathrm{r} 938$, pl. XXVI, fig. I et 3 ). Nous avons décrit aussi les articles en banane, fréquents dans les filaments axiaux des arbuscules de blastospores géantes. Nous avons indiqué que, malgré leur acido-résistance, ces éléments ne sont pas des chlamydospores, parce qu'ils n'ont pas de double contour et bourgeonnent sur place. Nous donnons de nouveaux exemples de secteurs et de blastospores géantes (fig. 5, pl. IV et fig. I et 2, pl. VIII).

4. Candida flareri. - Nous avons rencontré, dans un repiquage de cette espèce (souche type $583 \mathrm{~B}$ ), un bel exemple de secteurs plissés, clairs et sombres (fig. 7 , pl. IV) tels que nous les avons précédemment décrits ( $\mathrm{g} 38$, p. 499).

5. Candida pelliculosa. - Nous avons signalé (r938, p. 463, pl. XXIII, fig. 6) l'existence de fins secteurs sombres, rayonnant à la surface des colonies.

6. Candida albicans. - L'apparition de secteurs, dans les colonies de Candida albicans, doit ètre assez banale puisque nous l'avons observée chez nos souches $189,193,23$ r , 264, 296, 3o8, 332, 335, 374, 39 I, 4 го, 437 B, 453 B, 587 A et 587 B, 646 (fig. I, 2, 3, r5, pl. IV).

Ces secteurs sont plus ou moins développés, de couleur plus sombre, ivoire ou jaune sale, et tranchent sur le fond blanc de la colonie normale.

Ils semblent partir du fond de la colonie, car les parties blanches paraissent les recouvrir. D'autre part la vitesse de croissance ne doit pas être égale dans les secteurs blancs et les secteurs sombres; ces Annales de Parasitologie, T. XVIII, Ner 1-2-3. - 1941 . 
derniers dépassent en général le bord de la colonie et se terminent fréquemment sur leur bord libre par une couronne de filaments qui s'enfoncent dans le milieu, alors que, sur les parties blanches, les limites de la colonie sont nettement dessinées.

Ce sont les secteurs de cette espèce que nous avons particulièrement étudiés, mais les particularités que nous allons décrire se retrouvent dans les secteurs des autres espèces.

Morphologie microscopique des secteurs sombres. - $\mathrm{Si}$ on prélève séparément des parcelles de culture sur une partie blanche et sur un secteur sombre, on constate qu'aux différences de couleur et de vitesse de croissance correspond une différence morphologique si accentuée, si frappante, que nous avons d'abord cru qu'il s'agissait d'un mélange d'espèces.

Variante sombre de la souche 296 A (ovalis) (1). - Nous avons choisi cette souche pour étudier le phénomène, car il est chez elle très prononcé.

La partie blanche (fig. 2, pl. VI) est constituée par de petites levures légèrement ovales, isolées ou présentant un petit bourgeon.

$\mathrm{Au}$ contraire, dans les secteurs sombres (fig. I, pl. VI), on trouve de très grandes cellules ( 10 à $r 5$ fois plus grandes que celles des secteurs blancs), les unes de forme allongée, ayant quelquefois l'apparence de bouteilles pourvues d'un col, les autres ovales avec un prolongement en bec. Le contenu de ces éléments n'est pas homogène : on y voit parfois une masse arrondie assez bien délimitée.

Ces énormes différences morphologiques se retrouvent plus ou moins accusées chez toutes les souches présentant des secteurs sombres. Pourtant c'est en général la forme allongée qui prédomine dans les autres souches, la forme en bouteille étant beaucoup plus rare.

Réversibilité. - Nous avons pu observer la réversibilité des secteurs sombres à la forme normale blanche, au cours d'expériences entreprises pour séparer les deux espèces, à l'époque où nous pensions que l'apparition des secteurs sombres était due à un mélange.

Par une série de dilutions progressives, effectuées en partant des secteurs sombres, nous sommes arrivés à obtenir des colonies aplaties de la teinte du secteur sombre et formées de grandes cellules allongées, et, d'autre part, quelques colonies blanches typiques.

(1) La souche dite ovalis est l'ancien Mycotoruloides ovalis Langeron et Tálice $\mathrm{rg}_{9} 3$, qui, étudié de nouveau par Langeron et Guerra (rg38), s'est montré être un Candida albicans typique. Cette souche est intéressante à plusieurs points de vue : elle a été isolée à Coblentz, en 1927 , par le médecin principal Pichon, chez un soldat français de l'armée du Rhin, atteint de rhino-pharyngo-stomatite ; en outre, elle a attiré notre attention par la netteté de ses secteurs sombres et elle a été l'objet de nos premières recherches à ce sujet. 
Avec la souche ovalis 296 , nous avons pratiqué jusqu'à 9 séparations successives par la méthode de dilution.

Les colonies obtenues aux différentes étapes se maintenaient quelque temps " pures ", c'est-à-dire uniquement formées de grandes cellules pâles, allongées. Mais tòt ou tard on voyait apparaître de petites cellules rondes; le phénomène devenait même visible à l'œil nu. Au centre d'une colonie sombre, on apercevait un point blanc qui envahissait progressivement toute la colonie. Quelquefois, le point blanc se formait au centre de la colonie et on voyait à ce niveau des filaments s'enfoncer comme des racines dans la profondeur du milieu. On pouvait mème se demander si la coloration blanche n'était pas due à la plus grande épaisseur de la colonie qui augmentait l'opacité.

Cette réversibilité s'est manifestée toujours avec la mème intensité aux différentes étapes de nos séparations par dilution, sans que le milieu employé pour l'observation des cultures ait eu une influence quelconque.

Cultures sur lames montrant le mécanisme de la formation des secteurs sombres. - Parallèlement à nos expériences de dilution, nous avons voulu obtenir des cultures monocellulaires, de manière à trancher définitivement la question.

La technique employée a été la suivante. Nous avons d'abord fait une culture sur lame en partant d'une colonie très jeune de la variante sombre. Nous laissons la culture sur lame se développer pendant 8 jours. Nous sortons alors la lame et, avec une fine aiguille montée, nous prélevons, sous le microscope, la pointe d'un filament. On choisit ce filament bien dégagé, de sorte que son extrémité soit suffisamment éloignée de la strie d'ensemencement. Il est très facile ainsi de prélever des blastospores provenant d'un germe unique. Avec la colonie ainsi obtenue, on fait de nouvelles culiures sur lames. Nous avons pratiqué ainsi jusqu'à 5 " purifications " successives.

Or, même après ces " purifications ", le dimorphisme se manifeste. Il n'y a donc pas de mélange d'espèces ou de souches.

\section{Exemples de dimorphisme cellulaire dans les colonies à secteurs.}

I. Candida albicans souche ovalis 296 A. - La figure 2, planche VII, représente un point d'une lame de la variante sombre pure après isolement. Au milieu de microcolonies formées de cellules allongées du type sombre (on voit même en haut à droite une cellule en bouteille) on voit un rameau des plus typiques de Candida albicans 
avec verticilles globuleux de petites blastospores, appartenant manifestement à la forme normale blanche.

Un autre aspect (fig. I, pl. VII), obtenu au cours des purifications, montre la coexistence de la variante sombre (en bas, à gauche) avec la forme normale blanche qui donne une colonie isolée typique avec chlamydospores.

La figure 4, planche VIII, représente une portion d'un long filament, partant d'une strie sombre, qui donne, sur un même rameau, des verticilles de la forme normale blanche et des verticilles de la variante sombre. Cette microphotographie prouve définitivement qu'il ne s'agit pas d'un mélange de souches, mais d'un dimorphisme cellulaire réversible.

2. Candida albicans, souche $39_{1}$ A. - La figure 4, pl. VI, montre une strie de la variété sombre provenant d'une colonie purifiée par isolement monocellulaire. On voit s'entremêler les deux types de blastospores et surgir un rameau issu d'une grande cellule du type sombre. La figure 6, pl. VI, montre le mème phénomène avec une netteté frappante. On assiste ainsi à la naissance d'un secteur sombre, dont le début est représenté par l'arbuscule si bien isolé. Cette figure fait comprendre aussi avec quelle facilité on peut prélever à l'aiguille, sous le microscope, une des deux variétés de cellules.

3. Candida albicans, souche oosporoides ( $\mathrm{r}$ ) $\mathrm{n}^{\circ} 588_{7}$ B. - - La figure 5, pl. VI, montre une strie faite avec un secteur sombre avant isolement par culture monocellulaire. On voit le début d'un secteur sombre, formé de cellules allongées : c'est cette zone qui filamente la première et enfonce ses racines dans la partie blanche, formée de petites cellules arrondies fortement colorées.

7. Candida triadis. - Dans notre mémoire de 1938 (p. 455 et 476 , fig. I, pl. XXI) nous disions n'avoir jamais observé, pour cette souche, de variante membraneuse ou de secteurs clairs ou sombres. Depuis, nous en avons vu dans des colonies de 3 mois et plus, de souches d'autre provenance que notre souche type de $r_{932}$ (souche $58_{7} \mathrm{~A}$ ) : souches $79^{5}$ B, souche Peyre provenant de crachats, cas Brégier (fig. 8, pl. IV) ; $7^{53} \mathrm{~B}$, souche norvégienne du docteur E. Aaser, à chlamydospores (fig. Io, pl. IV); $663 \mathrm{~B}$, souche Douvier provenant de crachats (fig. I I, pl. IV); $948 \mathrm{~B}$, souche Henry provenant d'un poulet (fig. I6, pl. IV).

(I) La souche dite oosporoides est un Candida albicans typique, donnant des secteurs sombres. Cette souche nous a été adressée par le $\mathrm{D}^{\mathrm{r}} \mathrm{Zach}$, de Vienne, qui l'a isolée d'un cas d'onychomycose et l'a décrite en 1933 sous le nom de Blastoden. drion oosporoides. 
La figure 4, pl. VII représente une modification très curieuse que nous avons déjà observée dans les souches du type ovalis à secteurs sombres (fig. 4, pl. VIII) : c'est l'apparition de verticilles constitués par des blastospores baculiformes. Nous nous sommes demandé si C. triadis, qui présente aussi ce phénomène (fig. $4, \mathrm{pl}$. VII) et dont les blastospores sont grandes et allongées, ne serait pas simplement une variante sombre stabilisée de $C$. albicans. C'est d'autant plus probable que les seuls caractères nets, permettant de séparer $C$. triadis de C. albicans, sont : la couleur plus foncée, l'apparition assez tardive d'un voile muqueux et l'assimilation moins nette de l'urée. Or ces caractères sont ceux des colonies sombres de $C$. albicans.

Caractères biologiques des éléments des secteurs sombres. - Nous avons étudié les caractères biologiques des colonies sombres récentes, avant l'apparition des blastospores du type normal. Comme il était à prévoir, ces propriétés (fermentations, élection des sucres, assimilation de l'azote) sont à peu près identiques à celles des colonies blanches pures.

Toutefois, dans les milieux liquides, nous avons quelquefois observé l'apparition de larges anneaux muqueux chez les variantes sombres, mais ces anneaux sont transitoires. Nous avons de mème constaté le retour très rapide à la normale blanche des colonies sombres en milieu liquide.

\section{B. - Segteurs des colonies de torulopsoïdées}

Nous avons constaté l'apparition de secteurs dans un certain nombre de souches de Torulopsis et de Rhodotorula. Le plus souvent ces secteurs diffèrent à peine du reste de la colonie par leur coloration : ce sont plutôt des zones en relief ou en creux, par exemple chez Torulopsis aeria, T. laurentii, T. sphærica, T. neoformans souche 8r2 B (cas Pinet, service du Prof. Debré). Nous avons aussi une souche non déterminée de Rhodotorula, provenant du Maroc $\left(\mathrm{n}^{\circ} 258 \mathrm{M}\right)$, qui présente cette particularité. Mais c'est surtout chez Torulopsis pulcherrima (Lindner) Lodder qu'on peut observer de beaux secteurs blancs sur un fond rouge foncé ou des secteurs rouges sur fond blanc (fig. 14, pl. IV).

On sait que cette espèce produit généralement un pigment rouge foncé qui peut, soit diffuser dans la masse de la gélose en la colorant sur une étendue plus ou moins grande, soit imprégner les éléments levuriformes eux-mèmes qui prennent alors une teinte rouge foncé. M. W. Beijerinck $\left(\mathrm{r}_{9} \mathrm{I} 8\right)$ a élucidé la genèse de cette coloration; la 
levure produit un chromogène incolore qui, en présence de traces d'un sel de fer soluble et d'oxygène, donne par oxydation ce pigment très coloré. L'expérience est très facile à réaliser en ajoutant à la gélose environ o gr. o ro p. Ioo de citrate de fer ammoniacal (ou d'un autre sel soluble, chlorure ferrique, sulfate ferreux, etc.). Mais la pigmentation apparaît la plupart du temps spontanément parce que le glycose brut renferme presque toujours des traces de fer parmi ses nombreuses impuretés.

Cette espèce ( $\mathrm{I}$ ) est d'autant plus intéressante en mycologie médicale qu'elle a souvent donné lieu à des méprises d'autant moins excusables qu'elle est très facile à reconnaître, soit à son pigment si particulier, qui en fait un réactif très sensible du fer, soit à ses grosses cellules à paroi très épaisse et à inclusions lipidiques (levure à graisse de Beijerinck) qui présentent tous les caractères des chlamydospores (fig. 8, 10, 12, pl. V). En ce qui concerne ces éléments, on trouve généralement, en examinant les colonies, des éléments levuriformes dimorphes : les uns (blastospores) plus nombreux, ovalaires, petits $(2,5$ à $3 \mu)$; d'autres (chlamydospores) beaucoup plus volumineux $(3,5$ à $6 \mu)$, arrondis, à paroi très épaisse, avec une capsule mucilagineuse plus ou moins développée. Au centre de ces grosses cellules rondes se trouve un globule graisseux colorable par le soudan III et les autres colorants des lipides. En outre, on trouve encore dans les colonies des ébauches de filamentisation plus ou moins développées, formées de blastospores allongées, mais sans verticilles de blastospores arrondies (fig. 9, I2, I3, I 7, pl. IV, souche de Delft).

Parmi les méprises auxquelles nous faisions allusion plus haut, nous pouvons signaler :

I. Monilia castellanii S. Re ${ }_{92} 5$ ( $J l$ of trop: med. and hyg., XXVIII, r 925 , p. ${ }_{1}{ }_{7}$ ). - Ce champignon, isolé de cas de furonculose par Castellani, a d'abord été signalé par cet auteur en 1924 ( $J l$ of trop. med. and hyg., XXVII, I924, p. 326 ) sous le nom de Cryptococcus sp., puis a reçu de $\mathrm{S}$. Re le nom spécifique $M$. castellanii l'année suivante (1925). Castellani en a repris l'étude avec Jacono en I 933 , sous le nom de Torulopsis castellanii Castellani et Jacono. Dans toutes ces descriptions, le pigment rouge est mentionné (cf. Re $19_{25} 5$; Castellani et Jacono 1933 , pp. 3 г $2-3$ I 3 , pour le pigment et fig. 38 A pour les grandes cellules à inclusions lipidiques).

(I) Torulopsis pulcherrima est une levure banale des fleurs et des fruits. Beijerinck l'a trouvée sur les raisins, dans le miel (surtout le miel de bruyère), le nectar des fleurs (notamment des labiées du genre Lamium, la poussière obtenue en polissant les grains de malt, le jabot des bourdons, etc., etc. (Folia microbiologica, I, 1912, p. 75 ). 
Nous avons étudié trois souches de Monilia castellanii, provenant toutes trois du laboratoire de Castellani. Ce sont nos souches $672 \mathrm{~A}$, arrivée du Ross Institute en mars $1931 ; 785 \mathrm{~B}$, reçue fin décembre ${ }_{1} 9^{3} 7$ et $965 \mathrm{~B}$, du 15 avril 1939 . Toutes trois ont été entretenues sur gélose peptonée à $\mathrm{I}$ p. roo et glycosée à 2 p. Ioo et ont toujours donné la pigmentation rouge et les grosses cellules graisseuses caractéristiques (fig. I 2 , pl. V).

Monilia (ou Torulopsis) castellanii Re ${ }_{9} 9^{25}$ est donc en réalité Torulopsis pulcherrima (Lindner I $88_{7}$ ) Saccardo, levure très banale et sans pouvoir pathogène. La dénomination de Re doit donc tomber en synonymie et disparaître de la nomenclature des champignons parasites de l'homme.

Ce qui vient compliquer la question, c'est qu'une autre souche très différente a été nommée par Redaelli Cryptococcus castellanii. Cette souche, envoyée par Castellani en ${ }_{192} 6$ au Bureau Central de Baarn, a été étudiée par Mlle Lodder ( 1934, p. 40). Elle est toute différente du vrai castellanii et a donné sur carotte et sur milieu de Gorodkowa des ascospores du type Debaryomyces : elle s'est trouvée identique au D. matruchoti Grigoraki et Péju r92I, levure très banale, non pathogène et ne sécrétant pas de pigment rouge.

2. Cryptococcus interdigitalis Pollacci et Nannizzi r926. - La souche étudiée par Mlle Lodder (r934, pp. 144-146) s'est trouvée à peu près identique à Torulopsis pulcherrima (Lindner), avec quelques différences dans la croissance en moût de bière, suffisantes pour justifier la création d'une variété variabilis Lodder 1934 .

Il faut noter que la souche étudiée ne provenait pas directement des auteurs de l'espèce, mais avait été expédiée par le laboratoire Benedek.

Toutefois, dans la description originale de Pollacci et Nannizzi (I miceti patogeni, $\mathrm{V}, \mathrm{n}^{\circ} 44, \mathrm{r} 9^{2} 6$ ), il est expressément fait mention des grosses cellules à paroi épaisse, avec grande gouttelette graisseuse. Nous-mèmes avons retrouvé ces cellules, absolument typiques comme forme et dimensions, dans la préparation originale de Pollacci et Nannizzi que possède l'Institut de parasitologie de la Faculté de médecine de Paris et qui fait partie de la collection type de cent préparations vendues avec les exemplaires de $I$ miceti patogeni. Ces cellules y sont peu nombreuses, parmi une grande quantité de petites levures ovalaires de $2 \mu 5$ à $3 \mu$.

Il n'y a donc aucun doute sur l'identité réelle du Cryplococcus interdigitalis : cette espèce doit tomber en synonymie avec Torulopsis pulcherrima et disparaître de la mycologie médicale.

3. La mycothèque de l'Institut de parasitologie possède une $4^{\mathrm{e}}$ sou- 
che d'origine humaine ( $\mathrm{n}^{\circ} 6 \mathrm{2}_{2} \mathrm{~B}$ ) de Torulopsis pulcherrima, envoyée en janvier ${ }^{3} 9^{3} 7$ par le docteur F. Zach, sous le nom de Eutorulopsis Lunge ( $=$ des poumons) Ia Zach $\mathrm{r} 93 \mathrm{o}$, en provenance de la Clinique universitaire pour la syphilologie et la dermatologie à Vienne. Cette souche est aussi typique que les précédentes et présente tous les caractères macroscopiques et microscopiques du T. pulcherrima (fig. 8 et ro, pl. V). Le malade, des poumons duquel elle a été isolée, est mort d'une blastomycose vraie à Torulopsis neoformans : T. pulcherrima était donc, dans ce cas, un saprophyte banal.

\section{II. - Secteurs des colonies de levures ascosporées.}

Jusqu'ici, c'est surtout dans le genre Debaryomyces que nous avons observé la production très apparente de secteurs clairs ou sombres. Nous en avons rencontré aussi dans des colonies d'Hansenula anomala (souche 58I, collection Guilliermond) et de Zygopichia chevalieri (souche $6 \mathrm{I}_{7} \mathrm{~A}$, collection Guilliermond).

\section{A. - Segteurs chez les Debaryomyces}

Dans les descriptions des ouvrages classiques, il n'est fait mention que des éléments levuriformes et des asques monospores avec becs de copulation. Pourtant les Debaryomyces produisent une filamentisation exactement comme les Mycotorulö̈dées. Cette filamentisation est très facile à observer, soit autour des colonies géantes en tubes de gélose glycosée inclinée, soit dans les colonies développées sur lames.

Le pseudomycelium est toujours formé de blastospores comme chez les Candida : ces blastospores naissent normalement, à l'apex des articles du pseudomycélium, mais elles restent isolées ou forment seulement des chaînettes, sans constituer les verticilles et les glomérules si caractéristiques des Candida (pl. IX, fig. I-4). On obtient donc une ramification buissonnante et très touffue par suite de l'accumulation des blastospores qui se détachent et s'entassent autour des filaments. Les fig. 3, pl. VI, 3, pl. VII, 3, pl. VIII et I à 4, pl. IX donnent une bonne idée de cette filamentisation très caractéristique, qu'on peut déjà reconnaître en examinant les tubes par transparence, même si les colonies sont sèches (fig. 3 , pl. VII). Outre les blastospores, il se forme aussi une quantité plus ou moins grande de pseudoconidies de forme variable, arrondies, allongées ou stalagmoïdes, qui contribuent à donner à la filamentisation son aspect touffu et tourmenté caractéristique.

Nous avons pris, comme exemples de cette filamentisation, 
D. matruchoti, souche 5 r 4 A (souche du C. B. S. de Baarn); D. klöckeri, souche $574 \mathrm{~A}$ (= Debaryomyces B de Guilliermond et Cesari); D. guilliermondi, souche 55o (= Debaryomyces $K$ de Guilliermond et Cesari); Debaryomyces, souche 518 B (souche Novoty, érosion interdigitale du pied).

Les colonies à secteurs sont fréquentes et très nettes chez les Debaryomyces. Nous donnons comme exemples (fig. 2-6, 9, II, pl. V) D. hudeloi, souche 599 A, collection Guilliermond (= Atelosaccharomyces hudeloi de Beurmann et Gougerot r 9 Io, Dekker emend.) (fig. 3 et 4 , pl. V), puis une série de Debaryomyces dont nous n'avons pas encore fait la détermination spécifique : souche Colzol $n^{\circ} 417 \mathrm{~B}$ (fig. 6 et $\mathrm{II}, \mathrm{pl} . \mathrm{V})$, provenant de la bouche d'un individu sain servant de témoin (cette souche donne des secteurs blancs particulièrement nets); souche Néret $453 B$ (fig. 5 , pl. V), isolée d'un onyxis (grands secteurs blancs en relief); souche Guelaire $425 \mathrm{~B}$ (fig. 9, pl. V), à grands secteurs sombres, isolée d'un perionyxis typique après guérison; souche Labretonnière $55_{2} B$ (fig. 2, pl. V), isolée d'une lésion trichophytique, donnant aussi de grands secteurs sombres.

Notons en passant que tous ces Debaryomyces sont des saprophytes de la peau ou des muqueuses et que leur rôle pathogène est des plus douteux.

\section{B. - Secteurs chez d’autres levures ascosporées}

Nous avons observé des colonies à secteurs sombres chez Saccharomyces pastorianus (souche $540 \mathrm{~A}$, collection Guilliermond, colonie de 7 mois $1 / 2$ ); Hansenula anomala (souche 58 $\mathrm{r}$ A, provenant de la collection Guilliermond) (fig. I, pl. V) et à secteurs blancs chez Zygopichia (Zygosaccharomyces) chevalieri (souche $6{ }_{1} 7 \mathrm{~A}$, provenant de la collection Guilliermond). Cette dernière souche est très intéressante parce qu'outre les secteurs, elle présente une alternance très nette des phases $\mathrm{S}$ et $\mathrm{R}$; la colonie que nous représentons (fig. 7 , pl. V) a 15 jours; elle provient d'une colonie du type $\mathrm{S}$ de $\mathrm{I} 8$ jours, ensemencée en partant d'une colonie du type $\mathrm{R}$ très accentué de 3 mois $\mathrm{I} / 2$, issue elle-mème d'une colonie $\mathrm{S}$ de 4 mois.

\section{III. - Secteurs et colonies des phases $\mathbf{R}$ et $\mathbf{S}$}

Le cas du Zygopichia chevalieri nous amène à examiner les rapports possibles entre secteurs et phases $R$ et $S$.

Dans un précédent mémoire ( 1939 -1 940$)$, nous avons montré que 
ces phases correspondent à des variations dans la filamentisation. Tous les observateurs sont d'accord pour admettre que les colonies $\mathrm{R}$ sont membraneuses, par conséquent filamenteuses (pseudomycélium à articles allongés). Nous avons été plus loin dans l'analyse du phénomène et nous avons montré que la filamentisation normale des champignons levuriformes s'accompagnait de deux autres types : d'une part de très longs filaments non cloisonnés, produits par les blastospores suivant un processus très spécial, auquel nous avons donné le nom de blastèse; d'autre part des chaînettes de pseudoconidies très développées chez certaines espèces telles que Candida tropicalis.

L'étude de colonies développées sur lames gélosées nous a permis de suivre ces phénomènes en employant des souches où ils sont particulièrement nets. Nous sommes arrivés à saisir le début de la phase $\mathrm{R}$ à partir d'une seule cellule de la phase $\mathrm{S}$. Nous pensons avoir démontré que, chez les levures, l'alternance de ces deux phases est un processus normal, dont nous avons étudié les facteurs morphogénétiques, et non une dissociation irréversible. De grandes analogies nous portent à croire que ces conclusions peuvent être appliquées aux colonies $\mathrm{R}$ et $\mathrm{S}$ des bactéries et notamment à celles du bacille tuberculeux.

L'apparition de secteurs clairs ou sombres sur les colonies de levures et de champignons levuriformes nous paraît être un phénomène de mème ordre, produit par des modalités de la filamentisation. La chose est très nette chez Candida parakrusei, dont les secteurs sont constitués par des filaments formés de blastospores géantes qui apparaissent parmi les filaments normaux. La question d'un mélange de souches ne peut ètre soulevée, car on voit ces blastospores géantes naître brusquement dans la continuité d'un filament normal à petites blastospores et, inversement, des verticilles de petites blastospores sortir de rameaux à grosses blastospores.

Chez Candida albicans, la figure I, pl. VI, montre la tendance à l'allongement que présentent les éléments des secteurs sombres, contrastant avec les petits éléments arrondis des colonies normales (fig. 2, pl. VI). Des phénomènes analogues sont très nettement représentés par les figures 4-6, pl. VI, dans lesquelles on voit la tendance à l'allongement et à la filamentisation des cellules qui constituent les premières ébauches des secteurs sombres.

En dehors des Candida, toutes les colonies à secteurs que nous avons rencontrées chez des Torulopsö̈dées ou chez des levures ascosporées (Debaryomyces) appartiennent à des espèces qui filamentent. Cette filamentisation ne semble pas avoir été remarquée ni figurée, c'est pourquoi nous l'avons décrite et figurée chez Torulopsis pulcherrima et chez les Debaryomyces. Il s'agit d'ailleurs toujours d'un 
pseudomycélium formé de blastospores articulées bout à bout et ayant subi un allongement plus ou moins prononcé.

\section{RÉSUMÉ}

On remarque fréquemment, à la surface des colonies de champignons levuriformes, des secteurs clairs ou foncés. Ces secteurs ne semblent pas avoir encore fait l'objet d'une étude d'ensemble. Nous les avons observés chez sept espèces de Candida, un Rhodotorula, quatre Torulopsis (parmi lesquels T. pulcherrima est le plus remarquable), un Hansenula, un Zygopichia, un Saccharomyces et cinq Debaryomyces. A ce propos, nous décrivons la filamentisation des Debaryomyces qui semble avoir passé inaperçue, ainsi que celle de $T$. pulcherrima. Nous concluons en établissant un rapprochement entre l'apparition des secteurs et celle des phases $\mathrm{R}$ et $\mathrm{S}$, dont nous avons donné une étude critique dans un précédent mémoire. Dans l'un et l'autre cas, il s'agit d'un phénomène de filamentisation, luimême sous la dépendance de facteurs morphogénétiques que nous avons étudiés. En outre, dans les deux cas, ce phénomène est réversible, le retour au type normal étant à la fois spontané et facilement réalisable. Il importait de faire connaître l'existence et la signification de ces secteurs, pour mettre en garde contre la création d'espèces nouvelles, basées sur ces variations morphologiques.

\section{Bibliographie}

Beijerinck (M. W.). - Levures chromogènes. Nouvelle réaction biologique du fer, Arch. néerlandaises de physiol. homme et animaux, II, 1918, pp. 609-615. Voir aussi : Folia microbiologica, I, 1912, p. $7^{5}$.

Castellani (A.) et Jacono (I.). - Observations on fungi isolated from cases of blastomycosis cutis and blastomycosis pulmonalis in North America and Europe. Remarks on blastomycetin. Jl. of trop. med. and hyg., XXXVI, 1933, pp. 297-321 ; cf. pp. 312-313 Torulopsis castellanii (Re 1925).

Henrici (A. T.). - Molds, yeasts and actinomycetes. John Wiley and Sons, NewYork, I93o; cf. p. 205.

Langeron (M.) et Guerra (P.). - Nouvelles recherches de zymologie médicale. Ann. de paras., XVI, I938, pp. 36-84, $162-179,429-476,481-525$, pl. XIIXXXIII.

- Remarques sur le Candida stellatoidea (Jones et Martin rg38). Ann. de paras., XVII, 1939-1940, pp. 257-26o, pl. XVIII.

Valeur et nature des variations et dissociations de colonies chez les champignons levuriformes. Ann. de paras., XVII, 1939-1940, pp. 447-469, pl. XXX-XXXII.

- Orientation de la filamentisation des champignons levuriformes cultivés sur lames gélosées. Ann. de paras., XVII, r939-1940, pp. 580-589. 


\section{EXPLICATION DES PLANCHES IV-IX}

\section{Planche IV}

Secteurs des colonies de levures anascosporées (1).

Fig. 1. - Colonies à secteurs de Candida albicans, souche 587 B (Blastodendrion oosporoides Zach 1933), I936.

Fig. 2. - Colonies à secteurs de Candida albicans, souche $33_{1} \mathrm{~A}$, collection Sabouraud (oïdiomycose, cas Lacloche).

Fig. 3. - Colonies à secteurs de Candida albicans, souche $646 \mathrm{~B}$, provenant d'un ulcus de l'estomac, ${ }_{19} 37$.

Fıg. 4. - Colonie à secteurs pétaloödes de Candida guilliermondi, souche 782 B, souche du $\mathrm{D}^{r}$ Rivalier, Hòp. Saint-Louis, onychomycose cas Picard, décembre 1937 .

Fıg. 5. - Colonies à petits secteurs de Candida parakrusei, souche $43_{2} \mathrm{~B}$, hyperkératose plantaire, souche Guerra, Hòp. Saint-Louis, cas Canina, rg34.

Fig. 6. - Colonie à secteurs sombres de Candida pseudotropicalis, souche $496 \mathrm{~B}$, cas de sprue du $\mathrm{D}^{\mathrm{r}}$ Grigaut, 1935 .

Fig. 7. - Colonie à secteurs sombres du Candida flareri, souche 583 B, souche originale de Redaelli, 1936.

Fig. 8. - Colonie à grands secteurs sombres de Candida triadis, souche $795 \mathrm{~B}$, levure de crachats, souche du $\mathrm{D}^{\mathrm{r}}$ Peyre, cas Brégier, 1938.

Fig. 9, 12, 13, 17. - Filamentisation de Torulopsis pulcherrima, souche 886, C.B.S., Delft, 1937 . Culture de 4 jours sur gélose glycosée à 2 p. roo. Encre de Chine.

Fı́. ıo. - Colonie à secteurs de Candida triadis, souche 753 B (souche norvé-

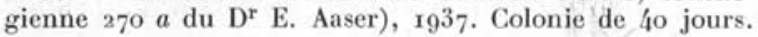

Fic. I1. - Colonie à secteurs de Candida triadis, souche $663 \mathrm{~B}$ bis, cas du $\mathrm{D}^{\mathrm{r}}$ Douvier, crachats, 1938. Colonie de ro2 jours.

Fıc. 14. - Colonies à secteurs rouges de Torulopsis pulcherrima, souche 965 BB, souche de Castellani, r939 (Torulopsis (Cryptococcus) castellanii). Colonies de 48 jours.

Fig. 15. - Colonie à secteurs sombres de Candida albicans, souche 308 B, souche Milochevitch, ig33. Colonie à sec.

Fıg. I6. - Colonie à secteurs de Candida triadis, souche $948 \mathrm{BB}$, poulet $95 \mathrm{~V}$ du $\mathrm{D}^{\mathrm{r}} \mathrm{Ch}$. Henry. Masse caséeuse péritonéale avec Aspergillus fumigatus.

\section{Planche V}

Secteurs des colonies de levures ascosporées.

Fıc. 1. - Colonie à secteurs sombres d'Hansenula anomala, souche 581 A (collection Guilliermond), 1930 .

Fic. 2. - Colonie à secteurs sombres du Debaromyces 552 $\mathrm{A}$ (Debaromyces $\mathrm{B} m$ de Cesari et Guilliermond), 1930 .

Fic. 3 et 4. - Colonies à secteurs blancs de Debaryomyces hudeloi, souche $599 \mathrm{~A}$ (collection Guilliermond), r93o. La colonie de la figure 3 est âgée de $9^{5}$ jours.

(I) Toutes les photographies de colonies à secteurs sont en grandeur naturelle. 
ANNALES DE PARASITOLOGIE

T. XVIII, Nos 1-2-3, 194'

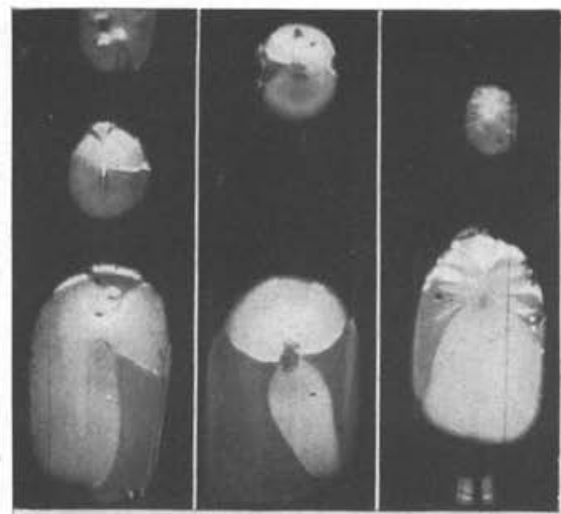

1

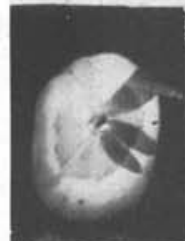

6

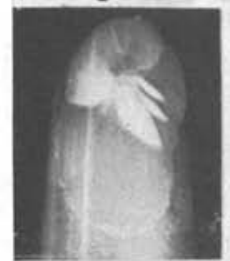

II

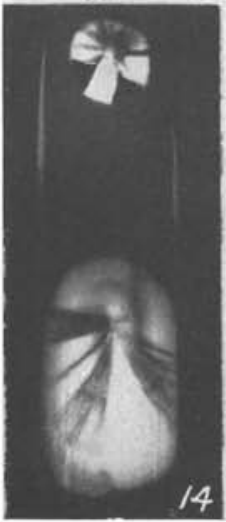

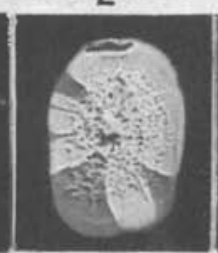

7
3

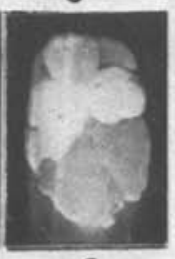

8

12
Planche IV

(Mémoire Langeron et Guerra)
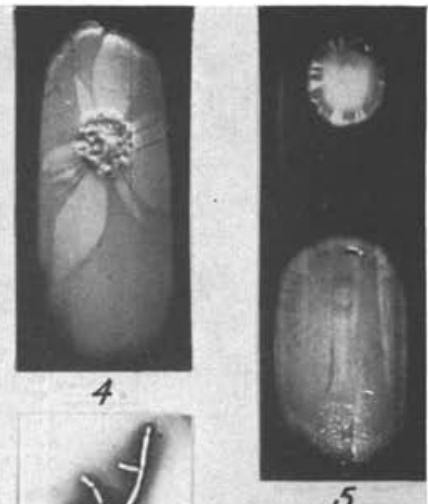

5

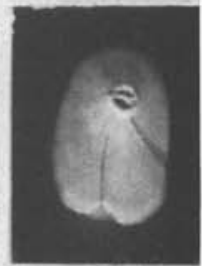

10

9
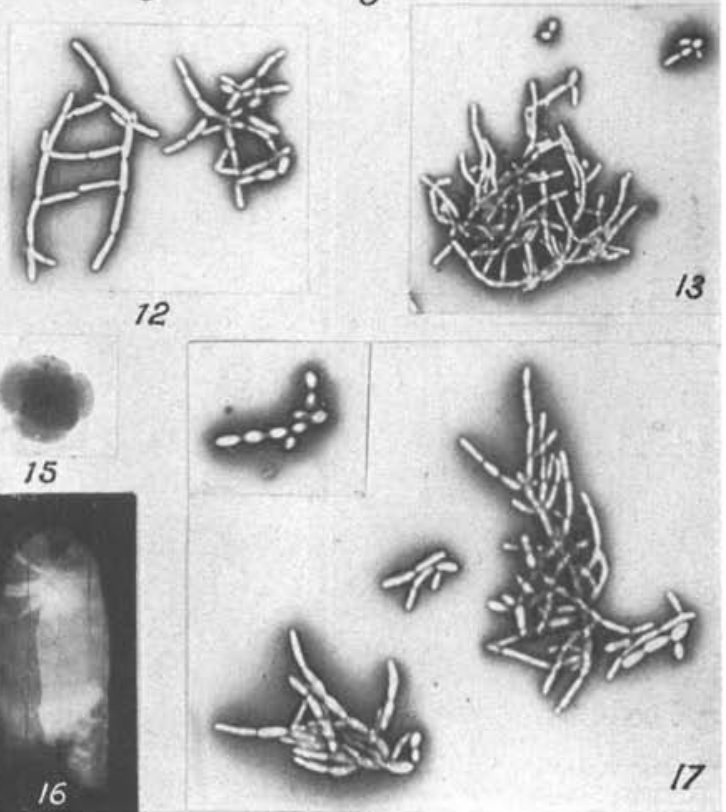

Masson et Cie, Édrteuns 
ANNALES DE PARASITOLOGIE

T. XVIII, Nos I-2-3, I94

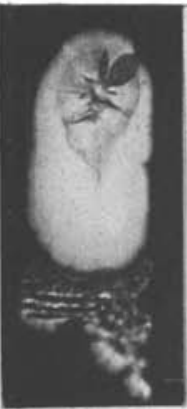

1

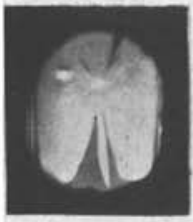

6

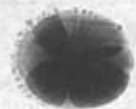

9

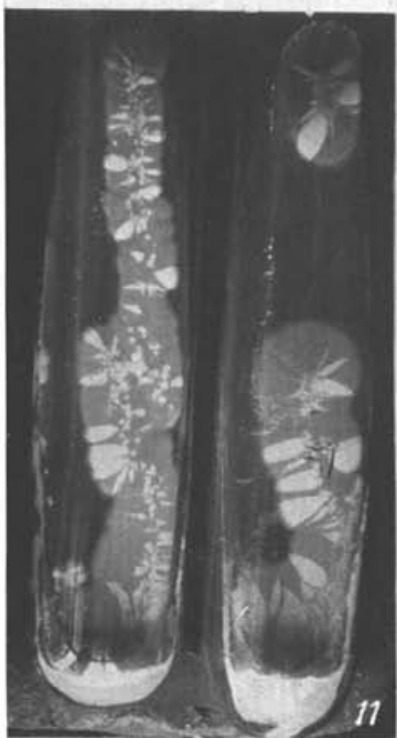

Planche V

(Mémoire Langeron et Guerra)

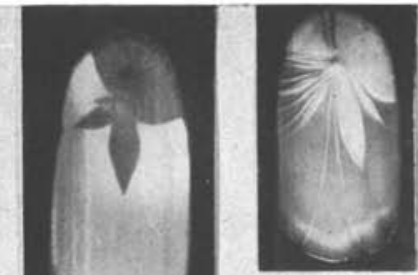

3

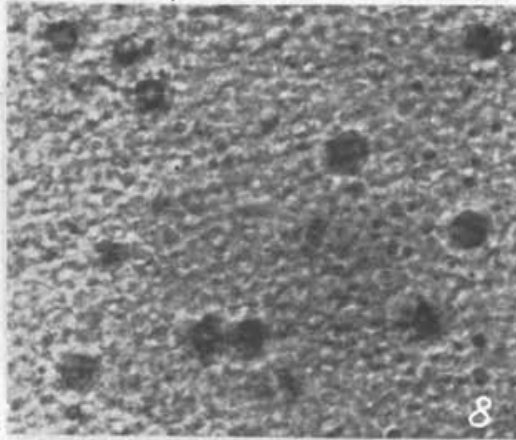

5itity

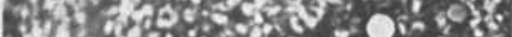

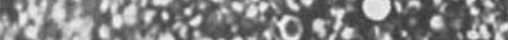

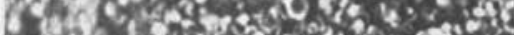

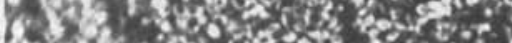

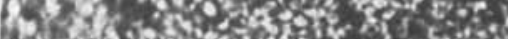

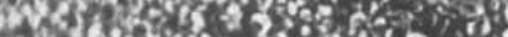

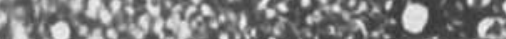

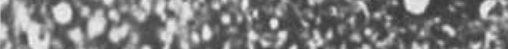

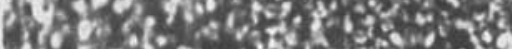

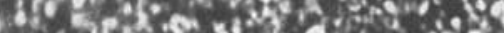

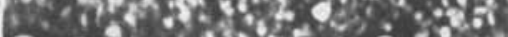

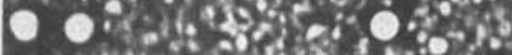

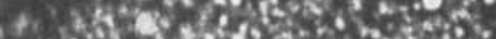

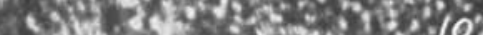

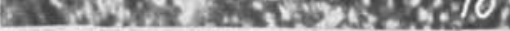

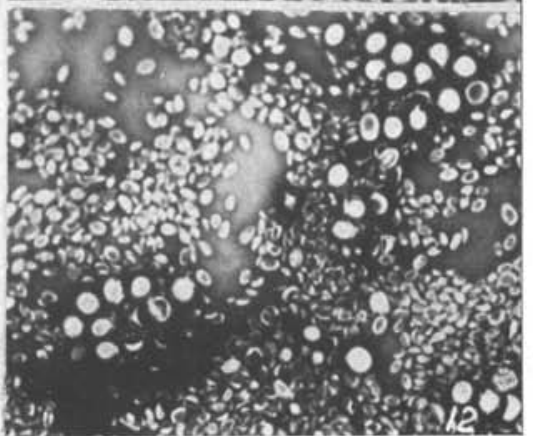



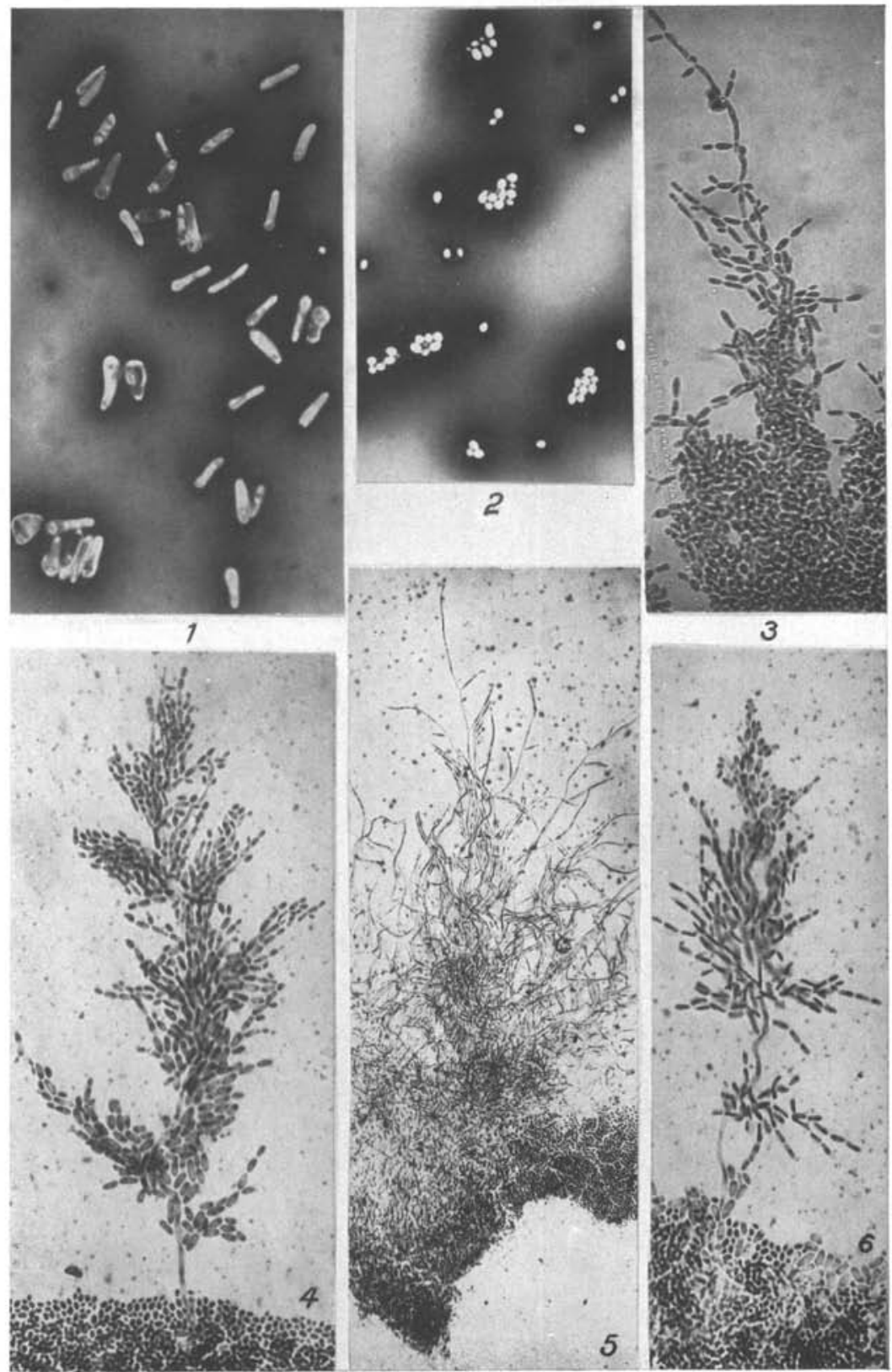

Masson et Cie, Éditeurs 

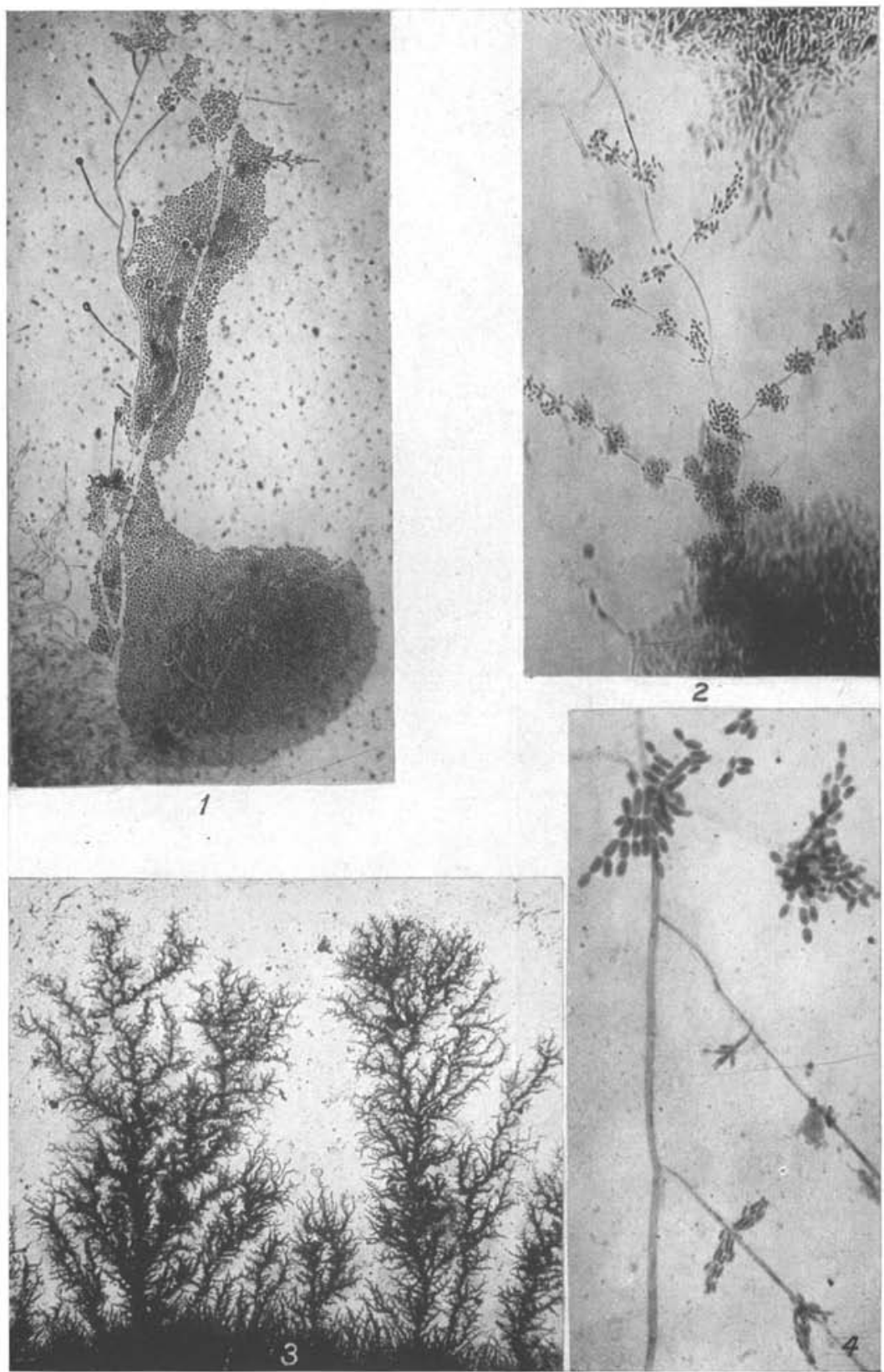

Masson et Cie, Édteuns 


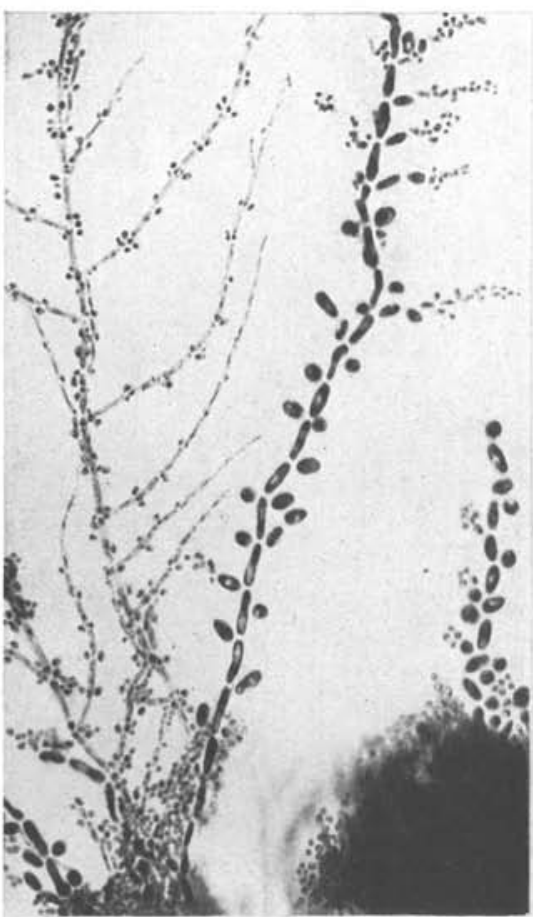

1

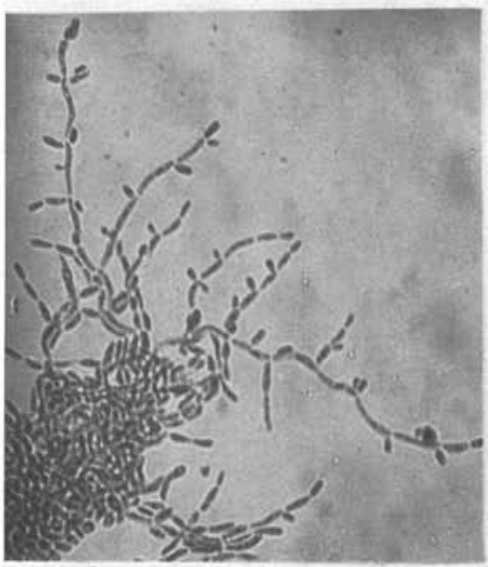

3

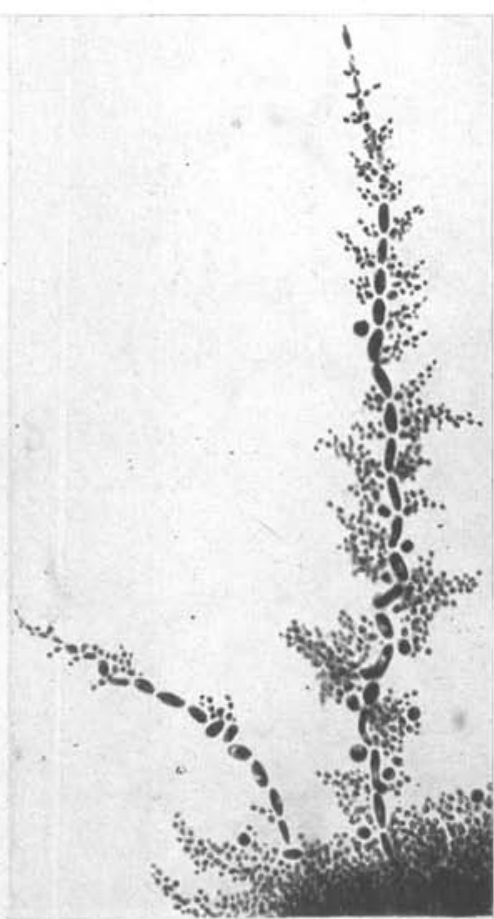

2

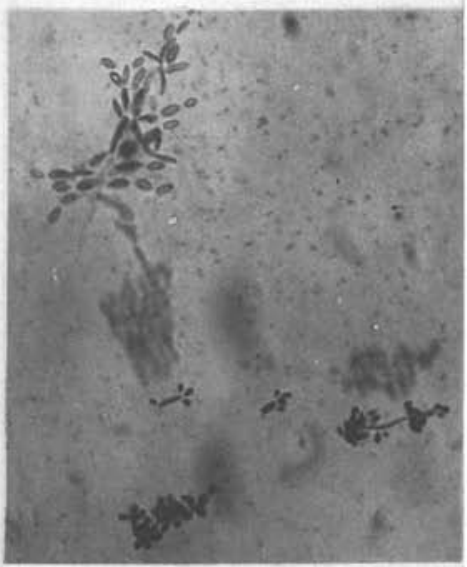

4 


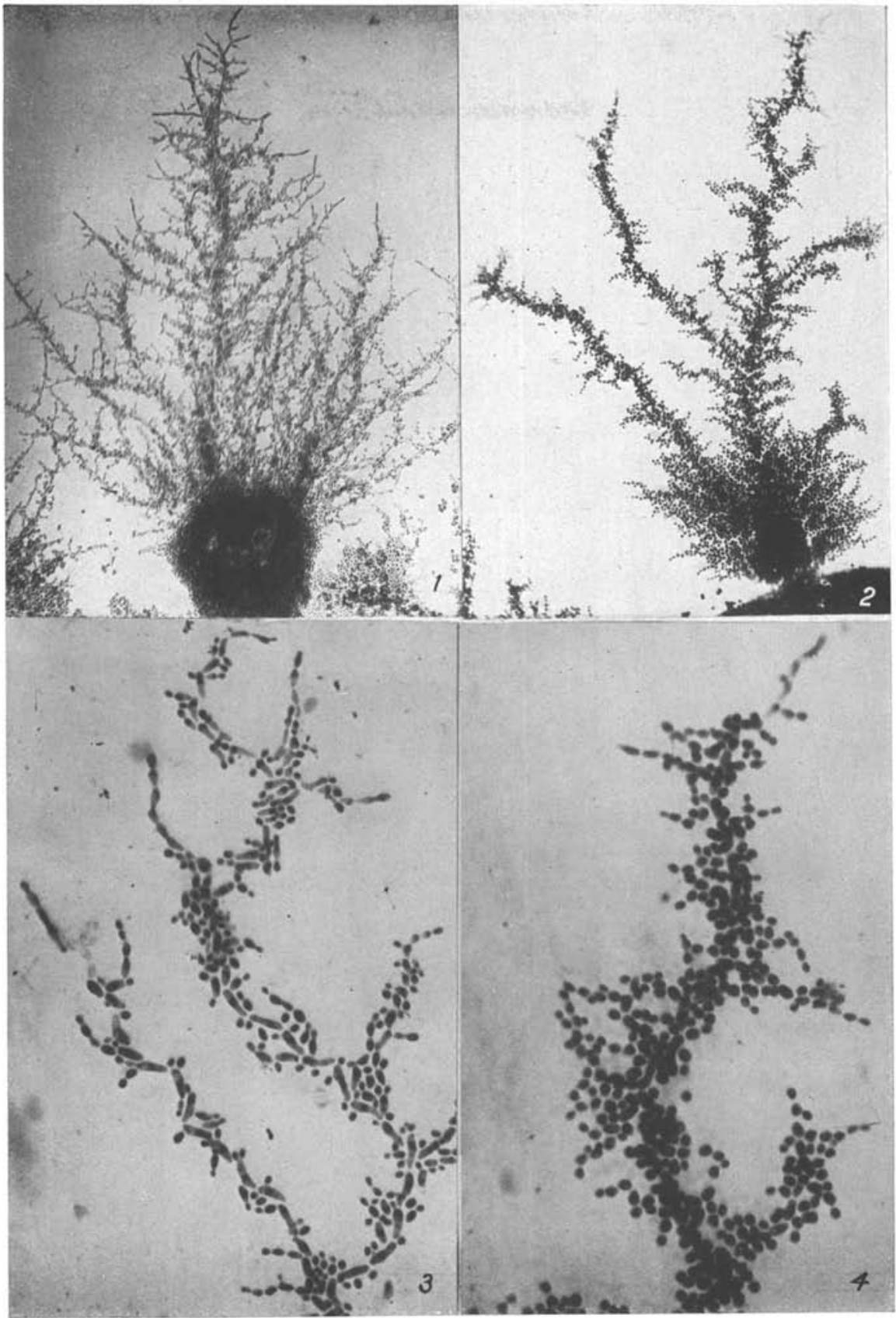


Fig. 5. - Colonie à secteurs du Debaryomyces n 435 B (souche Guerra, Hôp. SaintLouis, onychomycose, cas Néret), r934.

Frg. 6 et II. - Colonies à secteurs clairs et sombres du Debaromyces $4 \mathrm{r}_{7} \mathrm{~B}$ (souche Guerra, Hôp. Saint-Louis, cas Colzol, levure de la bouche), r934.

Fig. 7. - Colonie à secteurs de Zygopichia chevalieri, âgée de 15 jours, souche $6 \mathrm{r}_{7} \mathrm{~A}$ (collection Guilliermond), $\mathrm{rg} 3 \mathrm{o}$.

Fig. 8, ro, 12. - Blastospores et chlamydospores (levures à graisse de Beijerinck) de Torulopsis pulcherrima : fig. $\mathrm{I2}$, souche 672 A (Cryptococcus castellanii Re r925), souche Castellani du Ross Institute and Hospital, r931, encre de Chine; fig. Io, souche 620 B (Eutorulopsis I $a$ du $\mathrm{D}^{\mathrm{r}}$ Zach, Vienne), r937; encre de Chine; fig. 8, même souche 620 B, bleu au lactophénol Soudan.

Fic. 9. - Colonie à secteurs sombres (colonie à sec) du Debaromyces 425 B (souche Guerra, Hôp. Saint-Louis, r934, cas Guelaire).

\section{Plancie VI}

Dimorphisme des éléments des secteurs des colonies de levures.

Fic. r. - Eléments d'un secteur sombre d'une colonie de Candida albicans, souche ovalis $296 \mathrm{~A}$. Grandes cellules allongées ou en bouteille, avec col ou bec. Culture de 15 jours sur gélose glycosée à 2 p. roo. Encre de Chine.

Fıg. 2. - Éléments de la partie blanche, crémeuse, normale, de la même colonie. Culture de 6 jours sur gélose glycosée à 2 p. roo. Encre de Chine.

Fıc. 3. - Filamentisation d'un Debaryomyces, souche 5r8 B (souche Guerra, cas Novoti, érosion digitale du pied). Culture sur lame gélosée.

Frg. 4 et 6. - Origine des secteurs sombres des colonies du Candida albicans, souche 391 A (collection Sabouraud, oïdiomycose, cas Lacloche); éléments pris dans une culture en strie sur lame gélosée, faite en partant d'une colonie purifiée par culture monocellulaire de la variante sombre. La strie est constituée par un mélange des deux types de blastospores : les deux rameaux partent chacun d'une grande cellule du type sombre.

Fig. 5. - Origine de la variante sombre décelée dans une strie sur lame gélosée faite avec un secteur sombre avant isolement par culture monocellulaire. Candida albicans, souche $\mathrm{n}^{\circ} 587$ B (Blastodendrion oosporoides Zach 1933). La zone filamenteuse, à cellules allongées, est l'origine d'un secteur sombre; elle plonge ses racines dans la partie blanche, formée de petites cellules arrondies.

\section{Planche VII}

Dimorphisme des éléments des secteurs des colonies de levures.

Fı́. I. - Coexistence de la variante sombre à grands éléments allongés (angle inférieur gauche) et de la forme normale blanche à petits éléments arrondis, donnant une colonie isolée typique avec chlamydospores: Candida albicans, souche ovalis 296 A. Culture sur lame gélosée, colonie de 5 jours sur milieu PC ( 1 ).

(I) Le milieu PC est notre gélose à la pulpe de pomme de terre et de carotte (Langeron et Guerra, r938, p. 68).

Annales de Parasitologie, T. XVIII, Nos $1-2-3$. - $194 \mathrm{t}$. 
Fı́. 2. - Mème souche. Culture de 24 heures sur lame gélosée, milieu PC, de la variante sombre pure, dont on voit les grands éléments allongés en bas et en haut de la figure (remarquer en haut, à droite, une cellule en bouteille, isolée). $\mathrm{Au}$ milieu, un rameau très typique de la forme normale blanche, avec verticilles globuleux de petites blastospores.

Fig. 3. - Aspect typique de la filamentisation des Debaryomyces : bord desséché d'une colonie en tube de D. matruchoti, souche 514 A, C.B.S., Delft, 1930.

Fic. 4. - Verticilles normaux et verticilles à blastospores baculiformes coexistant sur le même rameau : Candida triadis, souche type $587 \mathrm{~A}$. Culture de 5 jours sur lame gélosée, milieu de Gorodkova.

\section{Planche VIII}

Dimorphisme des éléments des secteurs des colonies de levares.

Fıg. I et 2. - Coexistence de blastospores géantes et de blastospores normales dans une culture sur lame de 6 jours sur gélose glycosée à 2 p. 100 : Candida parakrusei, souche Ashford 34 I A, 1928. Ces blastospores géantes sont les éléments des secteurs jaunes marginaux (fig. 5 , pl. IV).

Fic. 3. - Filamentisation du type Mycocandida chez un Debaryomyces, souche 5r8 B (voir un autre point de la mème lame, fig. 3, pl. VI).

Fıc. 4. - Coexistence, sur le même filament, de verticilles de la variante sombre à grandes blastospores et de petits verticilles de la forme normale blanche : Candida albicans, souche ovalis $296 \mathrm{~A}$. Culture de la variante sombre pure sur lame gélosée, colonie de 5 jours sur milieu PC.

\section{Planche IX}

\section{Filamentisation des Debaryomyces.}

Voir aussi pl. VI, fig. 3 , pl. VII, fig. 3 et pl. VIII, fig. 3 .

Fig. 1. - Microcolonie, sur lame gélosée, de Debaryomyces matruchoti, souche 514 A, C.B.S., Delft.

Fı́. 2. - Microcolonie, sur lame gélosée, de Debaryomyces guilliermondi, souche 550 A (Debaryomyces $\mathbf{K}$ de Cesari et Guilliermond) (collection Guilliermond).

Fı́. 3. - Eléments des filaments du Debaryomyces klöckeri, souche 574 A (Debaryomyces B de Cesari et Guilliermond) (colleetion Guilliermond). Culture en strie sur lame gélosée.

Fı́. 4. - Éléments des filaments du Debaryomyces guilliermondi, sonche 55o A (partie de la fig. 2). 
Lodder (J.). - Die anaskosporogenen Hefen. Die Hefesammlung des "Centraalbureau voor Schimmelcultures ". Beiträge zu einer Monographie der Hefearten. II Teil. Erste Hälfte. Thèse Fac. Sc. Utrecht, 1934, 256 p.

Punkari. (L.) et Henricr (A. T.). - A study of variation in a chromogenic asporogenous yeast. $J l$. of bacteriol., XXVI, I933, pp. г25-138.

- Further studies on spontaneous variation of Torula pulcherrima. Jl. of bacteriol., XXIX, r935, pp. 259-267.

Re (S.). - Observations on the cultural and biochemical characters of Monilia castellanii (Cryptococcus castellanii) and Monilia macroglossiae (Cryptococcus macroglossiae). Jl. of trop. med. and hyg., XXVIII, 1925, pp. $317-319$.

Urвaci (E.) et ZACH (F.). - Generalisierte Torulose (europäische Blastomykose). Eine klinisch-botanische Studie. Arch. f. Dermat. $u$. Syphil., CLXII, I930-1931, pp. 40I-42I.

Institut de parasitologie de la Faculté de Médecine de Paris et station expérimentale de Richelieu (Directeur : Prof. E. Brumpl). section de mycologie (Chef de service : $D^{\mathrm{r}}$ Maurice Langeron). 